\title{
In-Medium Properties of Vector Mesons in a Transport Approach
}

\author{
Janus Weil* \\ Institut für Theoretische Physik, Universität Giessen, Germany \\ E-mail: janus.weiletheo.physik.uni-giessen.de
}

Kai Gallmeister

Institut für Theoretische Physik, Universität Giessen, Germany

E-mail:kai.gallmeister@theo.physik. uni-giessen.de

Ulrich Mosel

Institut für Theoretische Physik, Universität Giessen, Germany

E-mail: ulrich.mosel@theo.physik.uni-giessen.de

\begin{abstract}
We present dilepton spectra from $\mathrm{p}+\mathrm{p}$ and $\mathrm{p}+\mathrm{Nb}$ collisions at a kinetic beam energy of $3.5 \mathrm{GeV}$, which were simulated with the GiBUU transport model assuming different in-medium scenarios. We compare these spectra to preliminary HADES data and show that GiBUU can describe the data reasonably well. Our simulations indicate that the intermediate dilepton-mass region is sensitive to the $\mathrm{N}-\Delta$ electromagnetic transition form factor, which up to now is unmeasured in the time-like region.
\end{abstract}

XLIX International Winter Meeting on Nuclear Physics

24-28 January 2011

BORMIO, Italy

${ }^{*}$ Speaker. 


\section{Introduction}

While the vacuum properties of most hadrons are known to reasonable accuracy nowadays, it is a heavily debated question how these properties change inside nuclear matter. In particular, for the light vector mesons, various theoretical predictions regarding their in-medium properties have been suggested.

Among these expected in-medium effects, a so-called "collisional broadening" of the meson spectral function, due to inelastic collisions with the hadronic medium, is presumed. A second class of predictions claims that the vector meson masses will be shifted in the medium. These changes of the peak mass are connected to the partial restoration of chiral symmetry in the medium and have been studied via QCD sum rules. This effect has been claimed to be seen in experiments, but is still being discussed controversially. For a recent review on in-medium effects, see [1].

For studying in-medium effects, the more prominent hadronic decay modes of the vector mesons are unfavorable, since they are affected by strong final-state interactions with the hadronic medium - in contrast to the rare dilepton decay modes, which only feel the electromagnetic force. Therefore the latter are ideally suited to carry the in-medium information outside to the detector, undisturbed by the hadronic medium.

Experimentally, dilepton spectra from elementary nuclear reactions are being studied for example with the CLAS detector at JLAB, where photons with energies of a few $\mathrm{GeV}$ interact with nuclei [2], or by the E325 experiment at $\mathrm{KEK}$, where $12 \mathrm{GeV}$ protons are used as projectile [3]. Also, the HADES detector at GSI has an ambitious program for measuring dilepton spectra from $\mathrm{p}+\mathrm{p}$, $\mathrm{p}+\mathrm{A}$ and $\mathrm{A}+\mathrm{A}$ reactions [4]. On the side of the hadronic decays, most notably $\omega \rightarrow \pi^{0} \gamma$ is being investigated by the CB/TAPS group in photon-induced reactions at the ELSA accelerator [5].

In this paper, we apply the Gießen Boltzmann-Uehling-Uhlenbeck transport model (GiBUU) [6] to the $\mathrm{p}+\mathrm{p}$ and $\mathrm{p}+\mathrm{Nb}$ reactions studied by the HADES collaboration. We use GiBUU to generate dilepton events and pass them through the HADES acceptance filter, in order to compare our calculations directly to the experimental data measured by HADES.

\section{The GiBUU Transport Model}

Our tool for the numerical simulation of dilepton spectra is the GiBUU hadronic transport model, which provides a unified framework for various types of elementary reactions on nuclei as well as heavy-ion collisions [6]. This model takes care of the correct transport-theoretical description of the hadronic degrees of freedom in nuclear reactions, including the propagation, elastic and inelastic collisions and decays of particles. The GiBUU model is based on the Boltzmann-UehlingUhlenbeck equation,

$$
\left(\partial_{t}+\vec{\nabla}_{p} H \cdot \vec{\nabla}_{r}-\vec{\nabla}_{r} H \cdot \vec{\nabla}_{p}\right) f_{i}(\vec{r}, p, t)=I_{\text {coll }}\left[f_{i}, f_{j}, \ldots\right],
$$

which describes the space-time evolution of the one-particle phase-space density $f_{i}$ of a given particle species $i$ under the influence of a mean-field potential. The phase-space densities $f_{i}$ at time $t$ depend on the spatial coordinates $\vec{r}$ and the four momentum $p$. The right-hand side of the equation is given by the collision term $I_{\text {coll }}$, which describes collisions and decays of particles. The left-hand side, the so-called "Vlasov" part, describes the propagation of particles in a mean field, where $H$ is 
the relativistic one-particle Hamiltonian. In order to solve the BUU equation numerically, we rely on the test-particle ansatz. Here the phase-space densities are approximated by a large number of test particles, each represented by a $\delta$-distribution in coordinate and momentum space.

The propagation of particles with density-dependent spectral functions (usually referred to as "offshell propagation") poses a particular challenge. Our approach to this problem is based on the offshell equations of motion of test particles, as given in [7]. Such an off-shell treatment is necessary for including in-medium modifications of the spectral functions (e.g. collisional broadening of the vector mesons). The collisional width inside a nuclear medium of density $\rho$ can be related to the collision cross section $\sigma_{N X}$ with the nucleons in low-density approximation as

$$
\Gamma_{\text {coll }}=\rho\left\langle v_{\text {rel }} \sigma_{N X}\right\rangle,
$$

where $v_{\text {rel }}$ is the relative velocity and the brackets indicate an integration over the Fermi momentum of the nucleons. In general this collisional width will depend on the momentum of the involved particle $X$. However, a consistent treatment of such a momentum-dependent collisional width is not possible with the off-shell equations of motion mentioned above, as it will lead to superluminous test particles. Therefore we have to neglect the momentum dependence and keep only the linear density dependence

$$
\Gamma_{\text {coll }}=\Gamma_{0} \cdot \frac{\rho}{\rho_{0}},
$$

where $\rho_{0}=0.168 \mathrm{fm}^{-3}$ is the normal nuclear matter density. The value of $\Gamma_{0}$ should on average match the momentum-dependent width as obtained from the collision term. For the $\rho$ and $\omega$ mesons we typically use $\Gamma_{0}=150 \mathrm{MeV}$.

\section{The Collision Term / Elementary Cross Sections}

The collision term contains all sorts of scattering and decay processes: elastic and inelastic twobody collisions, decays of unstable resonances and even three-body collisions (which are only relevant at high densities). The two-body part of the collision term is separated into two different regimes in terms of the available energy $\sqrt{s}$ : a resonance model description at low energies and the Pythia string model at high energies.

For baryon-baryon collisions, the transition between the two is usually performed at $\sqrt{s}=2.6 \mathrm{GeV}$. There is a small window around this border $( \pm 0.2 \mathrm{GeV})$, where both models are faded linearly into each other in order to ensure a smooth transition. For meson-baryon collisions, the transition region lies at $\sqrt{s}=2.2 \pm 0.2 \mathrm{GeV}$.

The low-energy part is given by a resonance model [8], where basically all collision cross sections are assumed to be dominated by the excitation of nucleon resonances. The GiBUU model currently contains 61 baryon species (around 30 nucleon resonances plus strange and charmed baryons). The resonance model gives a good description of exclusive channels like one- and two-pion production as well as single $\eta$ and $\rho$ production.

At higher energies the resonance model breaks down. There we rely on the Monte Carlo event generator Pythia (v6.4.24) [9, 10], which is based on the Lund string model. Although PythiA clearly has its strengths in the high-energy regime, we use it down to energies of a few GeV. This 
works surprisingly well, as has recently been demonstrated for example by GiBUU's successful description of pion data measured by the HARP collaboration [11]. In the following, we will show that our approach also provides a rather good description of HADES dilepton spectra.

Since GiBUU is intended to be a multi-purpose generator, we follow the philosophy that PYTHIA is used with most of its default settings. It is obvious, however, that tuning a few selected parameters can lead to a much better agreement with specific experimental data; cf. appendix A for the parameter set used for the HADES calculations.

Furthermore, we had to extend PYTHIA in some more aspects. For instance PYTHIA's treatment of spectral functions is rather simplified and not sufficient for a proper description of dilepton spectra, which are very sensitive to the vector meson spectral functions. Therefore we had to replace PYTHIA's internal spectral functions by proper ones, because the former lack mass-dependent decay widths and are restricted to a narrow region around the pole mass. This also gives us the opportunity to introduce density-dependent spectral functions.

\section{Dilepton Decays and Form Factors}

In the GiBUU model the following dilepton decay modes are taken into account:

- direct decays, as $V \rightarrow e^{+} e^{-}$with $V=\rho^{0}, \omega, \phi \quad$ or $\quad \eta \rightarrow e^{+} e^{-}$,

- Dalitz decays, as $P \rightarrow e^{+} e^{-} \gamma$ with $P=\pi^{0}, \eta, \eta^{\prime} \quad$ or $\quad \omega \rightarrow \pi^{0} e^{+} e^{-} \quad$ or $\quad \Delta \rightarrow N e^{+} e^{-}$.

Most of them are treated similarly as in [12]. The leptonic decay widths of the vector mesons are taken under the assumption of strict vector-meson dominance (VMD),

$$
\Gamma_{V \rightarrow e^{+} e^{-}}(\mu)=C_{V} \frac{m_{V}^{4}}{\mu^{3}}
$$

with the constants $C_{V}$ listed in table 1 (taken from [13]).

\begin{tabular}{|c|c|c|c|}
\hline$V$ & $m_{V}(\mathrm{MeV})$ & $\Gamma_{e e}(\mathrm{keV})$ & $C_{V}=\Gamma_{e e} / m_{V}$ \\
\hline$\rho$ & 775.49 & 7.04 & $9.078 \cdot 10^{-6}$ \\
$\omega$ & 782.65 & 0.60 & $7.666 \cdot 10^{-7}$ \\
$\phi$ & 1019.455 & 1.27 & $1.246 \cdot 10^{-6}$ \\
\hline
\end{tabular}

Table 1: Dilepton-decay constants for $V \rightarrow e^{+} e^{-}$.

While the direct decay of the $\eta$ meson into a $\mu^{+} \mu^{-}$pair has been observed, for the corresponding $e^{+} e^{-}$decay only an upper limit of $\operatorname{BR}\left(\eta \rightarrow e^{+} e^{-}\right)<2.7 \cdot 10^{-5}$ is known [14]. However, the theoretical expectation from helicity suppression is still four orders of magnitude lower [15].

The Dalitz decays of the pseudoscalar mesons, $P=\pi^{0}, \eta, \eta^{\prime}$, are treated via the parametrization [16],

$$
\frac{d \Gamma_{P \rightarrow \gamma e^{+} e^{-}}}{d \mu}=\frac{4 \alpha}{3 \pi} \frac{\Gamma_{P \rightarrow \gamma \gamma}}{\mu}\left(1-\frac{\mu^{2}}{m_{P}^{2}}\right)^{3}\left|F_{P}(\mu)\right|^{2},
$$


with $\Gamma_{\pi^{0} \rightarrow \gamma \gamma}=7.8 \cdot 10^{-6} \mathrm{MeV}, \Gamma_{\eta \rightarrow \gamma \gamma}=4.6 \cdot 10^{-4} \mathrm{MeV}$ and the form factors

$$
\begin{array}{cl}
F_{\pi^{0}}(\mu)=1+b_{\pi^{0}} \mu^{2}, & b_{\pi^{0}}=5.5 \mathrm{GeV}^{-2}, \\
F_{\eta}(\mu)=\left(1-\frac{\mu^{2}}{\Lambda_{\eta}^{2}}\right)^{-1}, & \Lambda_{\eta}=0.676 \mathrm{GeV} .
\end{array}
$$

The above value of $\Lambda_{\eta}$ has been recently determined from the HADES data at $2.2 \mathrm{GeV}$ beam energy [17]. It should be noted that the form factors of the $\pi^{0}$ and $\eta$ Dalitz decays are sufficiently constrained by data, while the experimental constraints of the $\eta^{\prime}$ form factor are much weaker [16]. A VMD form factor for the $\eta^{\prime}$ Dalitz decay can be found for example in [18]. However, the $\eta^{\prime}$ contribution to the HADES dilepton spectra turns out to be practically insignificant. The parametrization of the $\omega$ Dalitz decay

$$
\begin{aligned}
\frac{d \Gamma_{\omega \rightarrow \pi^{0} e^{+} e^{-}}}{d \mu} & =\frac{2 \alpha}{3 \pi} \frac{\Gamma_{\omega \rightarrow \pi^{0} \gamma}}{\mu}\left[\left(1+\frac{\mu^{2}}{\mu_{\omega}^{2}-m_{\pi}^{2}}\right)^{2}-\frac{4 \mu_{\omega}^{2} \mu^{2}}{\left(\mu_{\omega}^{2}-m_{\pi}^{2}\right)^{2}}\right]^{3 / 2}\left|F_{\omega}(\mu)\right|^{2} \\
\left|F_{\omega}(\mu)\right|^{2} & =\frac{\Lambda_{\omega}^{4}}{\left(\Lambda_{\omega}^{2}-\mu^{2}\right)^{2}+\Lambda_{\omega}^{2} \Gamma_{\omega}^{2}}
\end{aligned}
$$

is adopted from $[19,12]$ with $\Gamma_{\omega \rightarrow \pi^{0} \gamma}=0.703 \mathrm{MeV}, \Lambda_{\omega}=0.65 \mathrm{GeV}$ and $\Gamma_{\omega}=75 \mathrm{MeV}$. We note here that the form factor of the $\omega$ Dalitz decay is also well-constrained by data [20].

For the $\Delta$-Dalitz decay, we use the parametrization from [21],

$$
\begin{aligned}
\frac{d \Gamma_{\Delta \rightarrow N e^{+} e^{-}}}{d \mu} & =\frac{2 \alpha}{3 \pi \mu} \Gamma_{\Delta \rightarrow N \gamma^{*}}, \\
\Gamma_{\Delta \rightarrow N \gamma^{*}} & =\frac{\alpha}{16} \frac{\left(m_{\Delta}+m_{N}\right)^{2}}{m_{\Delta}^{3} m_{N}^{2}}\left[\left(m_{\Delta}+m_{N}\right)^{2}-\mu^{2}\right]^{1 / 2}\left[\left(m_{\Delta}-m_{N}\right)^{2}-\mu^{2}\right]^{3 / 2}\left|F_{\Delta}(\mu)\right|^{2},
\end{aligned}
$$

where we neglect the electron mass. The electromagnetic N- $\Delta$ transition form factor $F_{\Delta}(\mu)$ is an issue of ongoing debate. Unlike the other semileptonic Dalitz decays, it is poorly constrained by data. At least at the real-photon point $(\mu=0)$ it is fixed by the decay $\Delta \rightarrow N \gamma$ to $\left|F_{\Delta}(0)\right|=3.029$, and also in the space-like region this form factor is well-constrained by electron scattering data on the nucleon. However, this form factor is basically unknown in the time-like regime, which is being probed by the $\Delta$ Dalitz decay.

From the theoretical side, many parametrizations are available for the space-like part, but most of them are not applicable in the time-like region. One of the few models which take care of the continuation to the time-like region is the two-component quark model given in [22].

\section{Dilepton Spectra from Elementary p+p Collisions}

It is very important to make sure that one understands the elementary reactions before moving on to heavier systems, which involve effects of the nuclear medium. Fortunately, HADES has also measured dilepton spectra from elementary $\mathrm{p}+\mathrm{p}$ reactions. These provide a base line for exploring the heavier nuclear systems. 

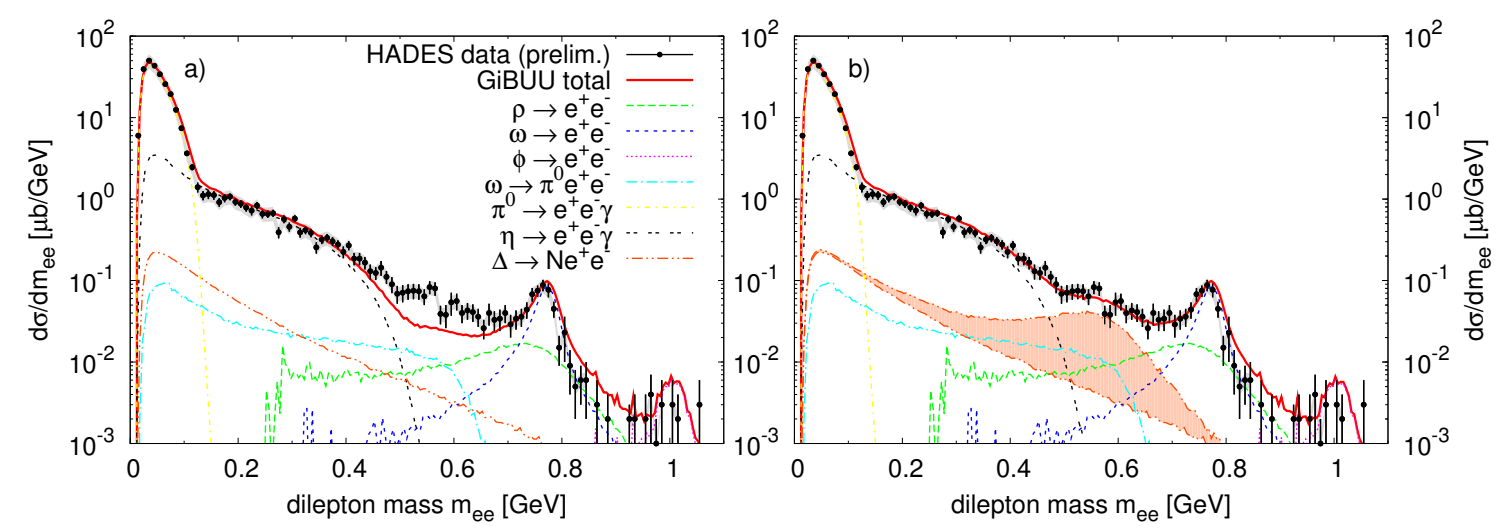

Figure 1: (Color online) Dilepton spectra for $\mathrm{p}+\mathrm{p} @ 3.5 \mathrm{GeV}$. a) Mass spectrum without $\Delta$ form factor, b) mass spectrum with $\Delta$ form factor.

Fig. 1 shows a comparison plot of a GiBUU simulation to HADES data [23] for a proton beam of $3.5 \mathrm{GeV}$ kinetic energy impinging on a fixed proton target. This setup corresponds to a centerof-mass energy of $\sqrt{s}=3.18 \mathrm{GeV}$. The theoretical results have been corrected for the HADES acceptance and reasonably reproduce the shape of the data over most of the mass spectrum. In the intermediate mass region around 500-600 MeV it seems like the inclusion of a proper transition form factor for the $\Delta$ Dalitz decay [22] (shaded area) is crucial for describing the data. Without such a form factor, the calculation strongly underestimates the experimental data in this region (by at least a factor of two). Another channel which could possibly contribute in the intermediate mass region is $\eta \rightarrow e^{+} e^{-}$. The current upper limit for the branching ratio of this decay would overshoot the HADES data by at least a factor of four. However, there is no $\eta$ peak visible in the data, and also the theoretical expectations from helicity suppression are still orders of magnitude below the current experimental limit. Therefore it is rather unlikely that the direct $\eta$ decay would give significant contributions to the HADES dilepton spectrum at 3.5 GeV.

It should be noted that the default PYTHIA parameters already give decent $\pi^{0}$ and $\eta$ production cross sections, while the vector meson production is overestimated quite a bit. This is cured by using the tuned parameters from appendix A.

In order to understand the underlying processes, it is not sufficient to consider only the mass spectrum. Other observables can give further insight into the reaction dynamics and can serve as a cross check for the validation of theoretical models. We choose to examine the transverse momentum and rapidity distribution in three different mass bins (see fig. 2):

1. The low mass bin $(m<150 \mathrm{MeV})$ is clearly dominated by the $\pi^{0}$ Dalitz channel, with some admixture of the $\eta$ Dalitz.

2. The intermediate mass range of $150 \mathrm{MeV}<m<550 \mathrm{MeV}$ mostly contains contributions of the $\eta$ and $\Delta$ Dalitz decays.

3. The high mass bin $(550 \mathrm{MeV}<m)$ is where the vector mesons are situated. 


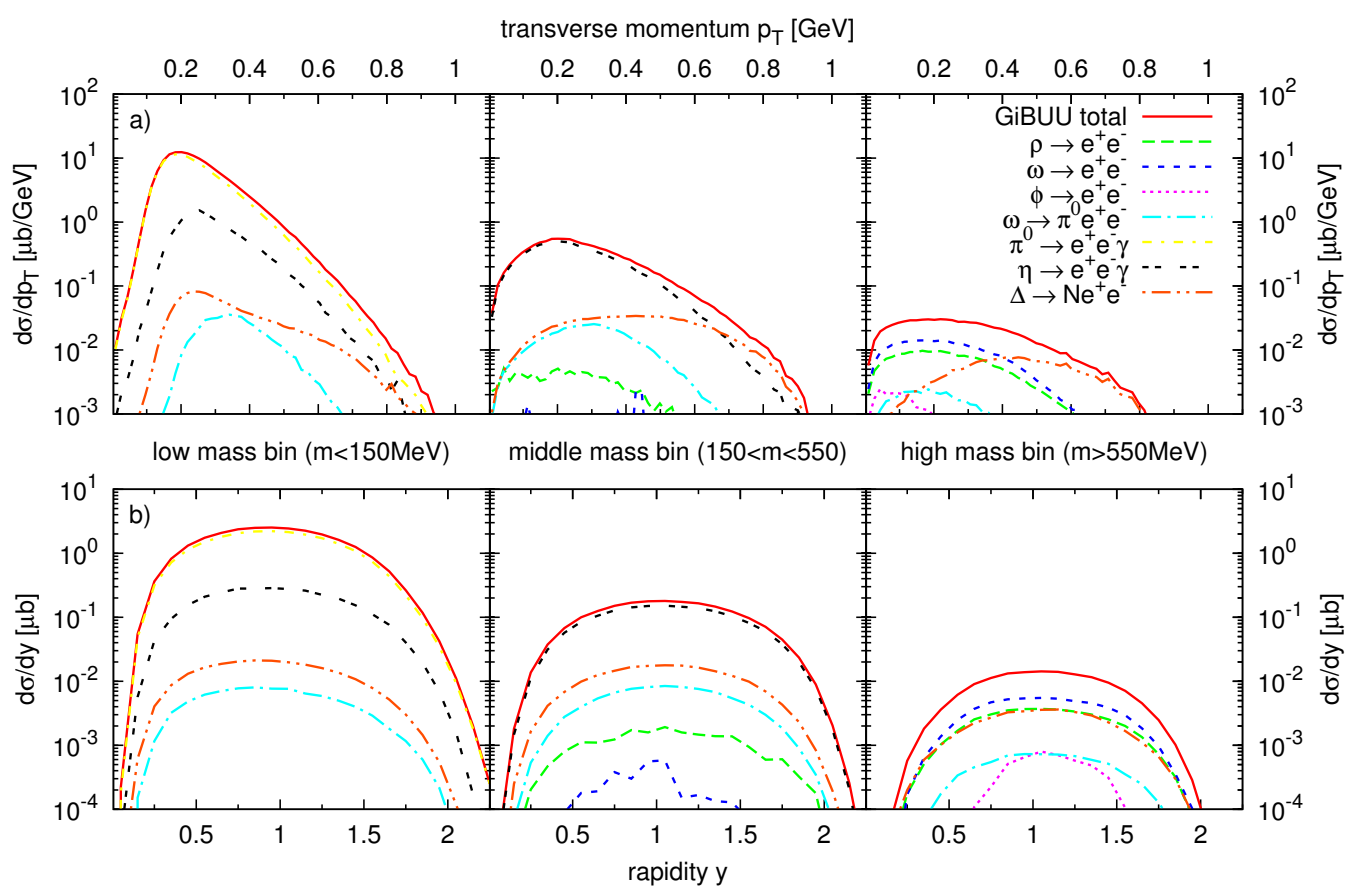

Figure 2: (Color online) a) Transverse momentum and b) rapidity spectra of dilepton pairs for three mass bins (with $\Delta$ form factor).

Distinguishing several mass bins is useful in order to separate the contributions of different channels. In all the three mass bins, we achieve a rather good agreement with preliminary data (not shown) [24], in transverse momentum as well as rapidity. With PYTHIA's default parameters, the $p_{T}$ spectra show a slight deviation from the data. The rapidity spectra turned out to be rather insensitive to the details of the reaction dynamics and to most of the parameters we modified. Their shape is mostly governed by the geometrical acceptance of the detector, which we apply to our simulations via the HADES acceptance filter (HAFT, version 2.0) [25, 26]. Therefore, the agreement in the rapidity spectra is mostly useful as a consistency check for the filtering process. The overall agreement proves to be quite good, although there are minor deviations at forward rapidity. The asymmetry of the deviation indicates that the deviation is indeed caused by acceptance filtering problems, since the unfiltered physical distributions are symmetric around mid-rapidity, and any asymmetries can only be introduced by the acceptance filter.

Within this level of agreement in the elementary $p+p$ collisions, we have a good baseline for studying in-medium effects in $\mathrm{p}+\mathrm{Nb}$, although the issue of the $\Delta$ Dalitz form factor is not completely settled.

\section{Dilepton Spectra from $\mathrm{p}+\mathrm{Nb}$ Collisions}

In $\mathrm{p}+\mathrm{Nb}$ reactions there will be couple of additional effects, compared to the elementary $\mathrm{p}+\mathrm{p}$ reactions. First of all, the primary $\mathrm{p}+\mathrm{N}$ collisions will be nearly identical, apart from binding effects and some Fermi smearing, but besides $\mathrm{p}+\mathrm{p}$ also $\mathrm{p}+\mathrm{n}$ collisions will play a role. Furthermore, the produced particles will undergo final state interaction within the $\mathrm{Nb}$ nucleus, and processes like 


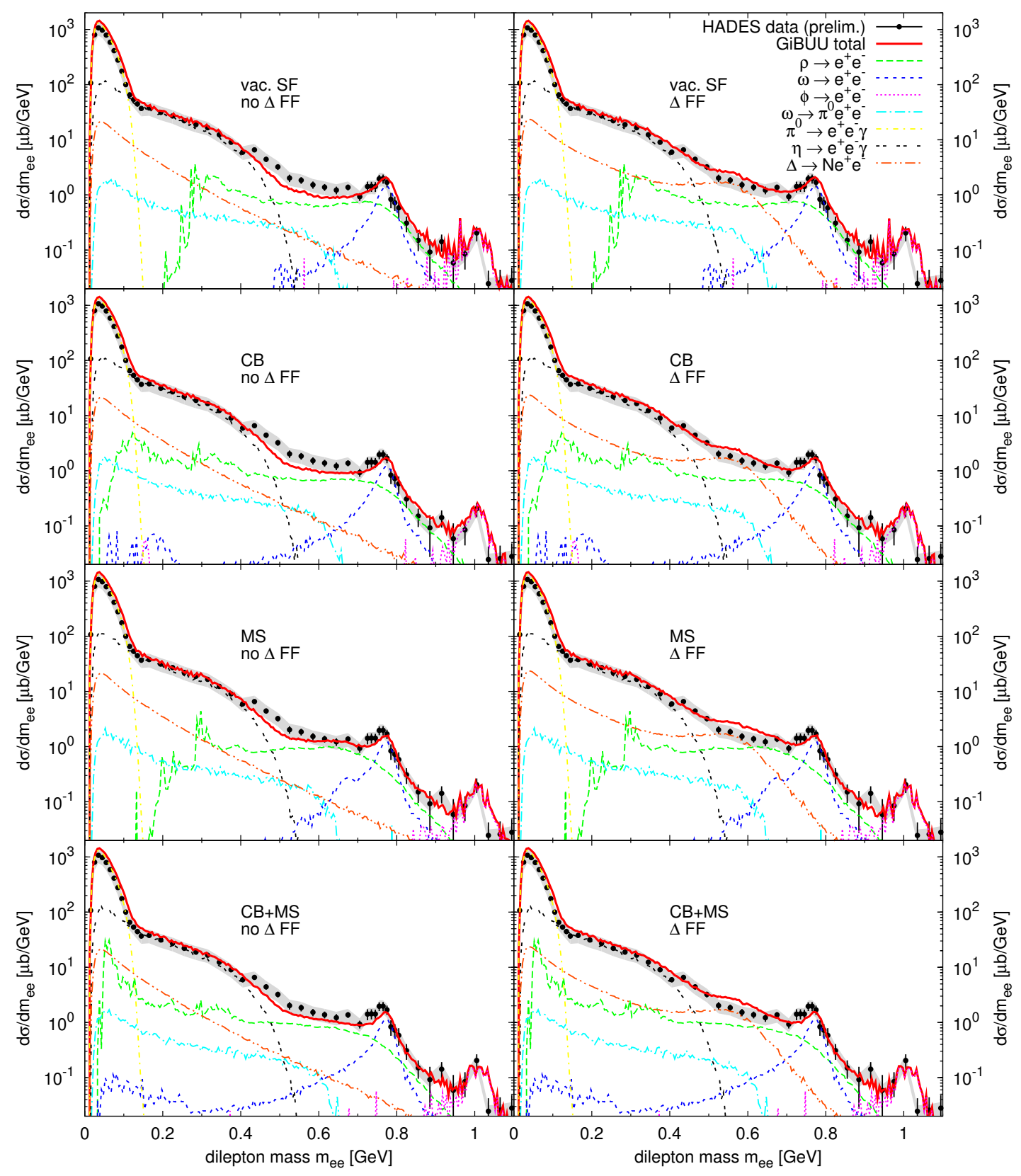

Figure 3: (Color online) Dilepton mass spectra for $\mathrm{p}+\mathrm{Nb} @ 3.5 \mathrm{GeV}$. Left column: without $\Delta$ form factor. Right column: with $\Delta$ form factor. From top to bottom: vacuum spectral functions for the vector mesons, collisional broadening, $16 \%$ mass shift, collisional broadening plus mass shift.

meson absorption and regeneration may become important. The secondary collisions will at average have lower energies than the primary $\mathrm{N}+\mathrm{N}$ collisions, therefore also the low-energy resonance part of the collision term will be involved. Finally, also the vector meson spectral functions may be modified in the nuclear medium.

Fig. 3 shows simulated dilepton spectra for $\mathrm{p}+\mathrm{Nb}$ collisions at $3.5 \mathrm{GeV}$ in various scenarios, compared to the data from [27]. The overall agreement is not quite as good as in the $\mathrm{p}+\mathrm{p}$ case. Already 


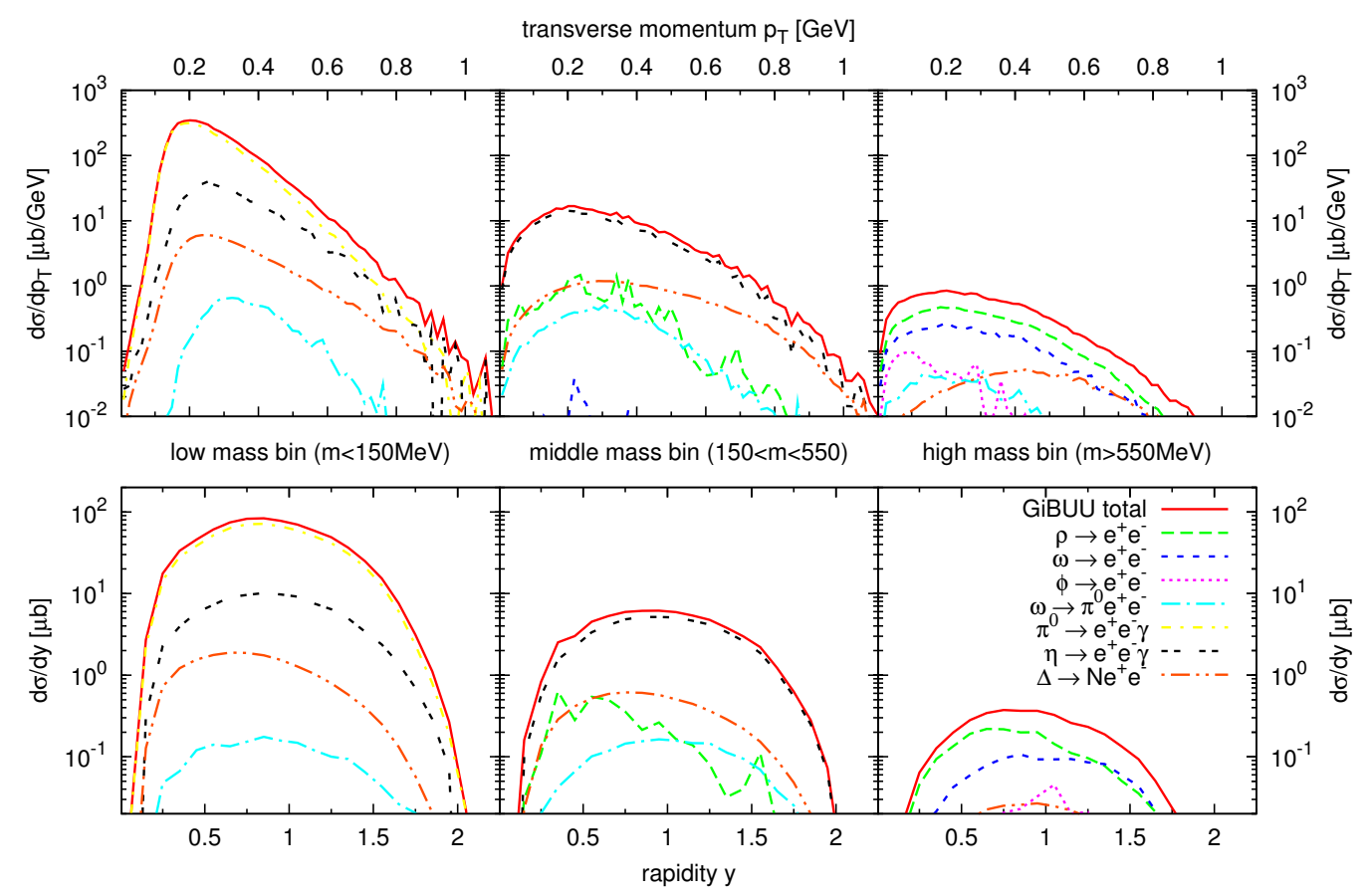

Figure 4: (Color online) $p_{T}$ and rapidity spectra for three mass bins: $m<150 \mathrm{MeV}, 150 \mathrm{MeV}<m<$ $550 \mathrm{MeV}$ and $550 \mathrm{MeV}<m$.

in the pion channel we slightly overestimate the data. This might have various reasons: too little absorption or too much secondary pion production in GiBUU, or even a normalization problem in the data.

According to [28], the data have been normalized by comparing charged pion spectra measured by HADES in $\mathrm{p}+\mathrm{Nb}$ to those measured by the HARP collaboration. However, the cross sections obtained by HARP had to be extrapolated to the slightly different beam energy and nuclear target of HADES. This procedure is responsible for most of the systematic error of the data (roughly 28\%), which is shown as a gray band in the figures.

Another striking feature of the $\mathrm{p}+\mathrm{Nb}$ system is that the simulation gets close to the data in the intermediate mass range, even without any $\Delta$ form factor. Including the form factor will slightly overshoot the data. It seems that most of the intermediate-mass gap observed in $p+p$ is filled up by low-mass $\rho$ mesons, which presumably are produced in secondary collisions. Even in $\mathrm{p}+\mathrm{p}$ collisions, one might already get a similar effect by describing $\rho$ meson production via resonance excitation, which could give stronger contributions in the low-mass part of the $\rho$ spectral function than PyTHIA's string fragmentation model.

The mass spectrum above $500 \mathrm{MeV}$ can receive further modifications from the inclusion of inmedium effects in the vector meson spectral functions. Fig. 3 shows a few typical in-medium scenarios: The first one includes a collisionally broadened in-medium width as described in chapter 2, while the second one assumes a pole mass shift according to

$$
m^{*}(\rho)=m_{0}\left(1-\alpha \frac{\rho}{\rho_{0}}\right)
$$


with a scaling parameter $\alpha=16 \%$. The third scenario combines both of these effects. The modifications introduced by these scenarios are roughly on the same order of magnitude as the systematic errors of the data. This fact, together with the discrepancy in the $\pi^{0}$ channel and the uncertainty of the $\Delta$ form factor, presently disables us to draw any hard conclusion on vector meson properties in cold nuclear matter from the HADES data.

The shape of the $p_{T}$ and rapidity spectra, depicted in fig. 4, show a similarly good agreement to preliminary data (not shown) [29] as in the $\mathrm{p}+\mathrm{p}$ case. The shown $p_{T}$ and rapidity spectra do neither include a $\Delta$ form factor nor any in-medium effects for the vector mesons and are not significantly sensitive to such modifications.

\section{Conclusions}

We have shown that the HADES data from elementary $\mathrm{p}+\mathrm{p}$ collisions at $3.5 \mathrm{GeV}$ kinetic beam energy can be described very well by the PYTHIA event generator with a few adjusted parameters, as employed by the GiBUU model. It was found that the intermediate mass region can be explained by including a VMD-like transition form factor for the $\Delta$ Dalitz decay. Also the $\mathrm{p}+\mathrm{Nb}$ reaction at the same beam energy is reasonably well described by the GiBUU transport model. However, a few discrepancies are left, which prevent us from drawing final conclusions on in-medium effects at this point. We infer that the HADES data at $3.5 \mathrm{GeV}$ can not be understood without fixing the open issue of the $\Delta$ Dalitz form factor.

\section{A. Pythia Parameters}

GiBUU relies on the PYTHIA event generator for elementary collisions at c.m. energies above 2.2 (2.6) $\mathrm{GeV}$ for meson-baryon (baryon-baryon) systems. PyTHIA's default parameters are tuned to rather high energies, just like most of the available parameter sets (called 'tunes' in PYTHIA). To apply PYTHIA successfully to collisions at HADES energies, we adjusted a couple of parameters. The following parameter set represents an attempt to create a 'HADES tune' for PYTHIA. A thorough description of all the parameters can be found in the PYTHIA manual [10].

\begin{tabular}{|c|c|c|c|}
\hline Parameter & $\begin{array}{c}\text { Default } \\
\text { value }\end{array}$ & $\begin{array}{c}\text { Professor } \\
\text { tune [30] }\end{array}$ & $\begin{array}{c}\text { Our } \\
\text { value }\end{array}$ \\
\hline PARJ(11) & 0.5 & 0.31 & 0.15 \\
PARJ(12) & 0.6 & 0.4 & 0.2 \\
PARJ(21) & 0.36 & 0.313 & 0.25 \\
PARJ(25) & 1.0 & 0.63 & 0.63 \\
PARJ(26) & 0.4 & 0.12 & 0.12 \\
PARP(91) & 2.0 & & 0.25 \\
\hline
\end{tabular}

Table 2: PythiA parameters tuned to HADES energies.

The most prominent modification is the suppression of the vector meson contributions by tuning down PARJ(11) and PARJ(12) by a significant amount. PARJ(25) and PARJ(26) are the parameters 
for suppressing the $\eta$ and $\eta^{\prime}$ contribution, which we adopt from the 'Professor' tune [30]. Moreover, the amount of transverse momentum has to be adjusted via PARP(91) and PARJ(21), which control the primordial $k_{T}$ and the $p_{T}$ from fragmentation, respectively.

\section{Acknowledgments}

We thank the HADES collaboration for providing us with the data and the HADES acceptance filter and for many fruitful discussions. Special thanks go to Tetyana Galatyuk, Anar Rustamov and Michael Weber. This work was supported by HGS-HIRe.

\section{References}

[1] S. Leupold, V. Metag, and U. Mosel, Hadrons in strongly interacting matter, Int.J.Mod.Phys. E19 (2010) 147-224, [arXiv:0907.2388].

[2] CLAS Collaboration, M. Wood et. al., Light Vector Mesons in the Nuclear Medium, Phys.Rev. C78 (2008) 015201, [arXiv: 0803.0492 ].

[3] M. Naruki, H. Funahashi, Y. Fukao, M. Kitaguchi, M. Ishino, et. al., Experimental signature of the medium modification for rho and omega mesons in 12-GeV p + A reactions, Phys.Rev.Lett. 96 (2006) 092301, [nucl-ex/0504016].

[4] HADES Collaboration, I. Frohlich et. al., Meson and di-electron production with HADES, Int.J.Mod.Phys. A24 (2009) 317-326, [arXiv: 0809.2764 ].

[5] CBELSA/TAPS Collaboration, M. Nanova et. al., Photoproduction of $\omega$ mesons on nuclei near the production threshold, Eur.Phys.J. A47 (2011) 16, [arXiv:1008.4520].

[6] "GiBUU website." http://gibuu.physik.uni-giessen.de.

[7] W. Cassing and S. Juchem, Semiclassical transport of particles with dynamical spectral functions, Nucl. Phys. A 665 (2000) 377-400, [nucl-th/ 9903070$].$

[8] S. Teis, W. Cassing, M. Effenberger, A. Hombach, U. Mosel, et. al., Pion production in heavy ion collisions at SIS energies, Z.Phys. A356 (1997) 421-435, [nucl-th/ 9609009 ].

[9] "Pythia website." http://projects.hepforge.org/pythia6.

[10] T. Sjostrand, S. Mrenna, and P. Skands, PYTHIA 6.4 Physics and Manual, JHEP 05 (2006) 026, [hep-ph/0603175].

[11] K. Gallmeister and U. Mosel, Production of charged pions off nuclei with $3 . . .30 \mathrm{GeV}$ incident protons and pions, Nucl. Phys. A826 (2009) 151-160, [arXiv: 0901.1770$].$

[12] M. Effenberger, Eigenschaften von Hadronen in Kernmaterie in einem vereinheitlichten Transportmodell. PhD thesis, Justus-Liebig-Universität Gießen, 1999. available online at http://theorie.physik.uni-giessen.de/.

[13] Particle Data Group Collaboration, K. Nakamura et. al., Review of particle physics, J.Phys.G G37 (2010) 075021.

[14] M. Berlowski, C. Bargholtz, M. Bashkanov, D. Bogoslawsky, A. Bondar, et. al., Measurement of eta meson decays into lepton-antilepton pairs, Phys.Rev. D77 (2008) 032004. 
[15] CLEO Collaboration, T. Browder et. al., A New upper limit on the decay eta $\rightarrow>$ e+ e-, Phys.Rev. D56 (1997) 5359-5365, [hep-ex/9706005].

[16] L. G. Landsberg, Electromagnetic Decays of Light Mesons, Phys. Rept. 128 (1985) 301-376.

[17] B. Spruck, Optimierung des Pionenstrahls zum HADES Detektor und Bestimmung des Eta-Formfaktors in Proton-Proton Reaktionen bei $2.2 \mathrm{GeV}$. PhD thesis, Justus-Liebig-Universität Gießen, 2008. http://geb.uni-giessen.de/geb/volltexte/2008/6667/.

[18] C. Terschlusen and S. Leupold, Electromagnetic transition form factors of light vector mesons, Phys.Lett. B691 (2010) 191-201, [arXiv:1003.1030].

[19] E. L. Bratkovskaya and W. Cassing, Dilepton production from AGS to SPS energies within a relativistic transport approach, Nucl. Phys. A619 (1997) 413-446, [nucl-th/9611042].

[20] NA60 Collaboration, R. Arnaldi et. al., Study of the electromagnetic transition form-factors in eta $\rightarrow>$ $m u+m u$ - gamma and omega $\rightarrow>$ mu+ mu- piO decays with NA60, Phys.Lett. B677 (2009) 260-266, [arXiv:0902.2547].

[21] M. I. Krivoruchenko and A. Faessler, Comment on Delta radiative and Dalitz decays, Phys. Rev. D65 (2002) 017502, [nucl-th/0104045].

[22] Q. Wan and F. Iachello, A unified description of baryon electromagnetic form factors, Int.J.Mod.Phys. A20 (2005) 1846-1849.

[23] HADES Collaboration, A. Rustamov, Inclusive meson production in 3.5-GeV p p collisions studied with the HADES spectrometer, AIP Conf.Proc. 1257 (2010) 736-740.

[24] A. Rustamov, "private communications."

[25] "HADES website." http://www-hades.gsi.de.

[26] T. Galatyuk, "private communications."

[27] HADES Collaboration Collaboration, M. Weber, Dielectron spectroscopy in cold nuclear matter, Int.J.Mod.Phys. A26 (2011) 737-740.

[28] M. Weber, Dielektronen-Spektroskopie in kalter Kernmaterie. $\mathrm{PhD}$ thesis, Technische Universität München, 2001. available online at http://mediatum.ub.tum. de/ node?id=1007264.

[29] M. Weber, "private communications."

[30] A. Buckley, H. Hoeth, H. Lacker, H. Schulz, and J. E. von Seggern, Systematic event generator tuning for the LHC, Eur. Phys. J. C65 (2010) 331-357, [arXiv: 0907 . 2973]. 